\title{
Diagnostic approach to Clostridium difficile infection
}

\author{
Chetana Vaishnavi
}

Received: 10 July 2010 /Accepted: 28 July 2010 /Published online: 18 August 2010

(C) Indian Society of Gastroenterology 2010

Clostridium difficile is recognized as the primary cause of hospital-acquired colitis in patients who receive antibiotics that alter their normal flora. $C$. difficile-associated disease (CDAD) is a growing nosocomial and public health problem with mortality up to $25 \%$ in frail elderly people [1]. The majority of hospitalized patients infected by $C$. difficile are asymptomatic carriers, who serve as silent reservoirs for continued $C$. difficile contamination of the hospital environment. Among all the risk factors, inclusive of the host and the environmental factors, antibiotics are the most important ones. Surgical patients comprise $55-75 \%$ of all patients with $\mathrm{CDAD}$ due to the fact that perioperative prophylaxis requires the use of antibiotics [2,3]. However, other drugs such as immunosuppressives, chemotherapeutics and proton pump inhibitors may also be important risk factors. The arrival of a mutant hypervirulent $C$. difficile bacterial strain, NAP1/BI/ 027 (North American PFGE type I/ restriction endonuclease analysis BI/ribotype 027) with 16-23 times higher levels of toxin production has increased the incidence of more severe clinical conditions like pseudomembranous colitis (PMC), megacolon and perforation [4]. Additionally, the recognition of community-acquired CDAD signals the presence of several risk factors.

Fecal lactoferrin assay [5, 6] performed simultaneously with C. difficile toxin assay can help rule out asymptomatic carriage of $C$. difficile. Pathogenic strains of $C$. difficile produce two high molecular weight toxins - A and B which bind to specific receptors on the luminal aspect of the colonic epithelium. Another toxin produced, known as binary

C. Vaishnavi $(\bowtie)$

Department of Gastroenterology,

Postgraduate Institute of Medical Education and Research,

Chandigarh 160 012, India

e-mail: cvaishnavi@rediffmail.com

e-mail: cvaishnavi@sify.com toxin CDT, has not been found to be essential for eliciting CDAD.

Toxin A initially disrupts the villus tips of the intestinal epithelium followed by damage to the brush border membrane. It also elicits the production of various cytokines and neurokinins that play an important role in pathogenesis. The eventual denuding of the mucosa, accompanied by extensive neutrophil infiltration, results in massive inflammation. The fluid response is partly an outcome of the damage to the intestinal epithelium. Toxin A also acts as a cytotoxin, leading to disruption of the tight junctions of the intestinal epithelium, and induces cell rounding which results in detachment of the cell from the basement membrane, followed by apoptosis. This brings about a rapid loss of resident cells such as macrophages, $\mathrm{T}$ cells and eosinophils.

The cytotoxic activity of toxin B is 1,000-fold more potent than that of toxin A. After toxin A has bound to the receptor initiating the damage, toxin $\mathrm{B}$ joins in and gains access to the underlying tissue and results in a number of non-specific in vitro responses. This includes disorganization of the actin filaments, loss of intracellular potassium, decrease in the level of protein and nucleic acid synthesis. The presence of toxin $\mathrm{A}^{-} \mathrm{B}^{+}$strains among clinical isolates documents that toxin $\mathrm{B}$ by itself may also be pathogenic in humans, as it is also a potent inflammatory enterotoxin for human intestine.

The clinical presentations in increasing order of severity include asymptomatic carriage, colitis without pseudomembrane formation, PMC and fulminant colitis with catastrophic transmural inflammation and myonecrosis. The most severe forms are the least common. Clinically, the disease can be diagnosed by symptoms of explosive watery, green, foul-smelling or bloody diarrhea accompanied by abdominal cramps. When benign diarrhea occurs with antibiotic use, no leukocytosis is seen. But in severely 
ill patients white blood cell counts of 20,000 per $\mathrm{mL}$ or greater maybe observed. At times, patients may experience occult colonic bleeding, and rarely may develop frank hematochezia. Other common manifestations include high fever, nausea, anorexia, malaise, dehydration and delirium. Hypoalbuminemia of $3.0 \mathrm{~g} / \mathrm{dL}$ or lower maybe observed in severely ill patients [7] where ascites maybe the only presenting manifestation of PMC. CDAD may present as fulminant colitis in approximately $3 \%$ of patients and account for most of the serious complications including perforation, prolonged ileus, megacolon and death [8]. Patients with ileus may have minimal diarrhea resulting in accumulation of secretions in the dilated, atonic colon.

Approximately $15-20 \%$ of CDAD patients relapse following successful treatment with vancomycin or metronidazole [9]. This condition is manifested by the sudden reappearance of diarrhea and other symptoms usually within a week of stopping treatment. Patients who relapse once are at an even greater risk of further relapses. Relapse is generally not related to antibiotic resistance because in some patients re-infection can occur with the same or different strain. The small bowel and the appendix may also act as reservoirs of $C$. difficile spores that enter the colon and result in relapse.

Pseudomembranous colitis is the classic manifestation of full-blown CDAD but as it appears late in the disease, it is not present always. When present, it appears as multiple yellow-white friable plaques, a few centimeters in size, attached to the underlying mucosa. About $10 \%$ cases of PMC go undetected when only sigmoidoscopy is done; the distribution of the membrane is patchy and it is more likely to occur in the proximal colon initially. Edema, blurring of the vascular pattern and thickening and blunting of the haustral folds may also be present. When PMC has been established, biopsy is confirmatory but not essential. Biopsy is essential when the mucosa appears inflamed, friable, granular or hemorrhagic and PMC has not been detected. Delay in the diagnosis of PMC could be lethal due to development of toxic megacolon or perforation. But endoscopy should be avoided in patients with paralytic ileus or colonic dilatation because of the risk of perforation. It is better reserved for special situations, such as when the patient is seriously ill and the results of rapid but not highly sensitive non-invasive tests are negative or delayed and CDAD is strongly suspected. However, it should be noted that other disorders may also produce pseudomembranes.

Pseudomembranous colitis can sometimes be diagnosed by computed tomographic (CT) scan when diarrhea is absent but abdominal pain, fever and leukocytosis occur. The risk of perforation and precipitation of megacolon deters a barium enema examination. $\mathrm{CT}$ scan findings are not pathognomonic of PMC, but it prompts strong consideration for initiation of specific therapy. It is most useful in PMC cases localized to the proximal colon and may reveal colonic distension, thickening, pericolonic inflammation, or free air. The patient may also have dilated small intestine with air-fluid levels mimicking intestinal obstruction or ischemia or pseudo-obstruction.

Clostridium difficile grows on selective media such as cefoxitin, cycloserine, fructose agar providing a low cost method. But culture is dependent upon the presence of spores or viable vegetative cells. The procedure is cumbersome and requires several days for results. Moreover it requires a follow up toxin testing as only about a third of the colonized isolates produce toxin. Culture nevertheless is useful for epidemiological and antibiogram studies during outbreaks.

C. difficile toxins can be detected in the fecal samples using tissue culture assay; this has been regarded as the gold standard, as it can detect as little as $1.0 \mathrm{pg}$ of toxin B. The disadvantage of tissue culture is the difficulty in maintenance of cell cultures and the procedure being expensive and time-consuming. False negative results can occur in stored samples due to toxin degradation or by delay in transportation or by medication. In fact, a negative cytotoxicity assay does not completely rule out $C$. difficile as the cause of diarrhea as $30 \%$ of patients maybe missed [10].

Polymerase chain reaction to detect toxin A or toxin B genes has sensitivity similar to cytotoxin testing but because it will detect even low numbers of $C$. difficile organisms that can be present in healthy individuals, it may give rise to wrong diagnosis.

Enzyme immunoassay (EIA) to detect toxin A or both toxins A and B in stool samples is widely used the world over. It has a sensitivity up to $90 \%$ and specificity up to $100 \%$. The advantage of EIA is predominantly the speed with which results are obtained, roughly two and a half hours. However, the high cost per single test may necessitate batching of samples. In this issue of the Journal, Modi and co-workers [11] report a retrospective review of C. difficile toxin testing. They calculated the unrefrigerated handling time for stool specimens by recording the stool collection time by nursing staff and receiving time of the specimens in the microbiology laboratory. They concluded that during unavoidable circumstances, stool specimens can be tested even if unrefrigerated for up to $13 \mathrm{~h}$ after collection. But one must bear in mind that room temperature in tropical countries is higher than that in the USA and the use of unrefrigerated specimens for toxin detection may not be acceptable everywhere. Moreover the global epidemic $C$. difficile strain, known to be a hyperproducer of toxins, might be responsible for sustained presence of toxin in stool specimens in countries like USA; this has not yet been reported in India. However in cases such as in PMC with infrequent diarrhea, the stool sample, even if unrefrigerated for a longer period of time, may become life saving at times. 
In conclusion, clinical suspicion for $C$. difficile infection is important because stool assays for diagnosing CDAD are not widely available. Wherever available it is fraught with inherent problems and therefore diagnosis may be missed or delayed.

Testing of stool from patients without clinical indications of $C$. difficile diarrhea is not only a redundant expense but may also complicate patient care if unnecessary antibiotic treatment is given. It should not be based on length of hospitalization but rather on the presence of clinically significant diarrhea with a history of antibiotic therapy or the presence of acute abdominal syndrome with little or no diarrhea.

\section{References}

1. Crogan NL, Evans BC. Clostridium difficile: an emerging epidemic in nursing homes. Geriatr Nurs. 2007;28:161-4.

2. Jobe BA, Grasley A, Deveney KE, Deveney CW, Sheppard BC. Clostridium difficile colitis: an increasing hospital-acquired illness. Am J Surg. 1995;169:480-3.
3. Gerding DN, Olson MM, Peterson LR, et al. Clostridium difficile associated diarrhea and colitis in adults: a prospective case controlled epidemiologic study. Arch Intern Med. 1986;146:95100.

4. Riley TV. Epidemic Clostridium difficile. MJA. 2006;185:133-4.

5. Vaishnavi C, Bhasin DK, Kochhar R, Singh K. Clostridium difficile toxin and fecal lactoferrin assays in adult patients. Microbes Infect. 2000;2:1827-30.

6. Vaishnavi C, Thapa BR, Thennarasu K, Singh K. Fecal lactoferrin assay as an adjunct to Clostridium difficile diarrhea. Indian $\mathbf{J}$ Pathol Microbiol. 2002;45:69-74.

7. Gebhard RL, Gerding DN, Olson MM, et al. Clinical and endoscopic findings in patients early in the course of Clostridium difficile-associated pseudomembranous colitis. Am J Med. 1985;78:45-8.

8. Rubin MS, Bodenstein LE, Kent KC. Severe Clostridium difficile colitis. Dis Colon Rectum. 1995;38:350-4.

9. Kelly CP, Pothoulakis C, LaMont JT. Clostridium difficile colitis. N Engl J Med. 1994;330:257-62.

10. Peterson LR, Kelly PJ, Nordbrock HA. Role of culture and toxin detection in laboratory testing for diagnosis of Clostridium difficile associated diarrhoea. Eur J Clin Microbiol Infect Dis. 1996;15:330-6.

11. Modi C, DePasquale JR, Nguyen NQ, Malinowski JE, Perez G. Does the handling time of unrefrigerated human fecal specimens impact the detection of Clostridium difficile toxins in a hospital setting? Indian J Gastroenterol. 2010;29. doi:10.1007/s12664-010-0040-1. 